\title{
Erratum to: NanoRiskCat: a conceptual tool for categorization and communication of exposure potentials and hazards of nanomaterials in consumer products
}

\section{Steffen Foss Hansen • Keld Alstrup Jensen • Anders Baun}

Published online: 30 June 2017

(C) Springer Science+Business Media Dordrecht 2017

Erratum to: J Nanopart Res16:2195 (2014)

DOI 10.1007/s11051-013-2195-Z

The original version of this article unfortunately contained two mistakes. There were discrepancies in Fig. 3 and Table 5 that have to be corrected as follows: (a) The "BCF $>50$ " which appear in Fig. 3 on page 9 has replaced with "BMF $>0.1$ "; (b) The red and yellow dot inserted in Table 5 for the environmental exposure potential and human health aspects of "Carbon nanotubes used to stiffen Babolat_NSTM Tour TennisRacket” has been replaced with a gray and red dot, respectively. 
The following shows the corrected Fig. 3

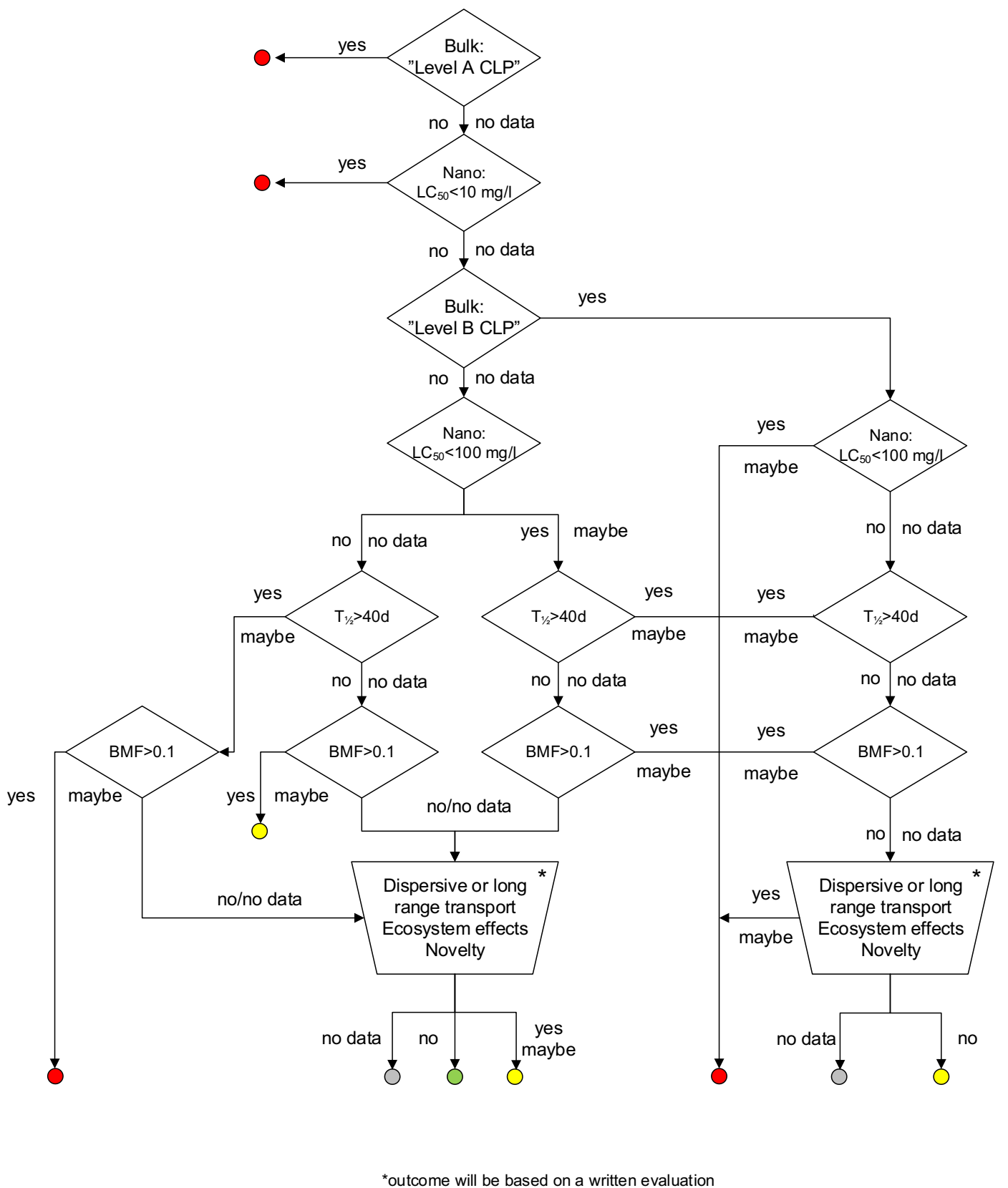

Fig. 3 Road-map for assigning an environmental hazard color code in NanoRiskCat. Red, yellow, and green indicate high, medium, and low indication of effect whereas gray indicates too limited data to make an assessment. Asterisk at least one "maybe" and the rest "no" or "no data" 
The following shows the corrected Table 5 .

Table 5 NanoRiskCat profiles for the eight different nanoproducts

\begin{tabular}{|c|c|c|c|c|c|c|c|c|}
\hline & \multirow[t]{2}{*}{ Use sentence } & \multicolumn{3}{|c|}{ Exposure } & \multicolumn{2}{|r|}{ Effect } & \multicolumn{2}{|c|}{ Hazard Sentences } \\
\hline & & $\begin{array}{l}\begin{array}{l}\text { Professional } \\
\text { end-users }\end{array} \\
\end{array}$ & Consumers & \begin{tabular}{|l|l} 
Environment \\
\end{tabular} & \begin{tabular}{|l} 
Human \\
health
\end{tabular} & \begin{tabular}{|l}
$\begin{array}{l}\text { Environmental } \\
\text { effects }\end{array}$ \\
\end{tabular} & Health hazard sentence & $\begin{array}{l}\text { Environmental } \\
\text { effect sentence }\end{array}$ \\
\hline 1. & $\begin{array}{l}\text { Nanosilver } \\
\text { used in } \\
\text { NANOVERTM } \\
\text { Cleansing Soap }\end{array}$ & (1) & (1) & 9 & (1) & $\theta$ & 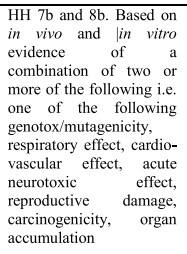 & $\begin{array}{l}\text { EE 2. Based on } \\
\text { nanospecific } \\
\text { LC50 or EC50 } \\
<10 \mathrm{mg} / 1\end{array}$ \\
\hline 2. & $\begin{array}{l}\text { C60 used in } \\
\text { Bardahl XTC } \\
\text { C60 5W40 } \\
\text { synthetic } \\
\text { special oil }\end{array}$ & (1) & (1) & (4) & (1) & (-) & 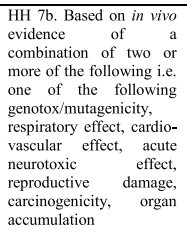 & $\begin{array}{l}\text { EE 2. Based on } \\
\text { nanospecific } \\
\text { LC50 or EC50 } \\
<10 \mathrm{mg} / 1\end{array}$ \\
\hline 3. & $\begin{array}{l}\text { Carbon } \\
\text { nanotubes used } \\
\text { to stiffen } \\
\text { Babolat } \text { NSTM }^{T M} \\
\text { Tour Tennis } \\
\text { Racket }\end{array}$ & (1) & (4) & (4) & (1) & (-) & $\begin{array}{l}\text { HH 1. Based on evidence } \\
\text { of HARN }\end{array}$ & $\begin{array}{l}\text { EE 3. Based on } \\
\text { possible or } \\
\text { confirmative } \\
\text { evidence of } \\
\text { nanospecific } \\
\text { L50 or EC50< } \\
100 \mathrm{mg} 71 \text { and } \\
\mathrm{T} 1 / 2>40 \mathrm{~d}\end{array}$ \\
\hline 4. & $\begin{array}{l}\text { TiO2 used in } \\
\text { BaByliss Pro } \\
\text { Stylist Tooks } \\
\text { Nano Titanium } \\
\text { Satin } \\
\text { Straightener }\end{array}$ & (1) & (1) & (4) & (1) & (2) & $\begin{array}{l}\text { HH 8b. Based on in vitro } \\
\text { evidence of } \\
\text { combination of two or } \\
\text { more of the following i.e. } \\
\text { one of the following } \\
\text { genotox/mutagenicity, } \\
\text { respiratory effect, cardio- } \\
\text { vasular effect, acute } \\
\text { neurotoxic enffect, } \\
\text { reproductive damage, } \\
\text { carcingenenicity, organ } \\
\text { accumulation }\end{array}$ & $\begin{array}{l}\text { EE 2. Based on } \\
\text { nanospecific } \\
\text { LC50 or EC50 } \\
<10 \mathrm{mg} / \mathrm{l}\end{array}$ \\
\hline 5. & $\begin{array}{l}25 \mathrm{~nm} \text { Zinc } \\
\text { Oxide } \\
\text { nanoparticles } \\
\text { used in UVa } \\
\text { and UVb } \\
\text { reflecting Solar } \\
\text { Rx SPF 30+ } \\
\text { Nano-Zinc } \\
\end{array}$ & (1) & (1) & (9) & (1) & $\theta$ & $\begin{array}{l}\text { HH 8b. Based on in vitro } \\
\text { evidence of a } \\
\text { combination of two or } \\
\text { more of the following i.e. } \\
\text { one of the following } \\
\text { genotox/mutagenenitity, } \\
\text { respiratory effect, cardio- } \\
\text { vascular effect, acute }\end{array}$ & $\begin{array}{l}\text { EE 1. Based on } \\
\text { bulk CLP } \\
\text { classification of } \\
\text { Acute 1 1 or } \\
\text { Chronic } 1 \text { or } \\
\text { Chronic 2 }\end{array}$ \\
\hline & $\begin{array}{l}\text { Oxide } \\
\text { Sunblock }\end{array}$ & & & & & & 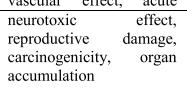 & \\
\hline 6. & $\begin{array}{l}\text { NanoCotz'TM } \\
\text { Eco-Clean } \\
\text { protective } \\
\text { silicon dioxide } \\
\text { coating }\end{array}$ & (1) & (4) & (9) & (1) & $\theta$ & $\begin{array}{l}\text { HH 7b. Based on in vivo } \\
\text { evidence of of a } \\
\text { combination of two or } \\
\text { more of the following i.e. } \\
\text { one of the following } \\
\text { genotox/mutagenicity, } \\
\text { respiratory effect, cardio- } \\
\text { vascular effect, acute } \\
\text { neurotoxic enffect, } \\
\text { reproductive damage, } \\
\text { carcinogenicity, organ } \\
\text { accumulation }\end{array}$ & $\begin{array}{l}\text { EE 2. Based on } \\
\text { nanospecific } \\
\text { LC50 or EC50 } \\
<10 \mathrm{mg} / \mathrm{l} \text {. }\end{array}$ \\
\hline 7. & $\begin{array}{l}\text { Gold used in } \\
\text { Chantecaille } \\
\text { Nano Gold } \\
\text { Energizing } \\
\text { Cream }\end{array}$ & (1) & (4) & (4) & (1) & (-) & $\begin{array}{l}\text { HH 13. Based on in vivo } \\
\text { evidence indicating at } \\
\text { least one hazard from } \\
\text { testing of the } \\
\text { nanomaterial }\end{array}$ & $\begin{array}{l}\text { EE 3. Based on } \\
\text { possible or } \\
\text { confirmative } \\
\text { evidence of } \\
\text { nanospecific } \\
\mathrm{L} 50 \mathrm{or} \text { EC } 50< \\
100 \mathrm{mg} / \mathrm{l} \text { and } \\
\mathrm{T} 1 / 2>40 \mathrm{~d}\end{array}$ \\
\hline 8. & $\begin{array}{l}\text { Unknown } \\
\text { nanomaterial } \\
\text { used in } \\
\text { Percenta AG } \\
\text { Nano Synthetic } \\
\text { Material } \\
\text { Sealant }\end{array}$ & (1) & (b) & (4) & (1) & $\theta$ & $\begin{array}{l}\text { HH 20. Based on the } \\
\text { identity of the } \\
\text { nanomaterial not being } \\
\text { disclosed or available, } \\
\text { which hampers any } \\
\text { human hazard evaluation }\end{array}$ & $\begin{array}{l}\text { EE 13. Based } \\
\text { on the identity } \\
\text { of the } \\
\text { nanomaterial } \\
\text { not being } \\
\text { disclosed or } \\
\text { available, which } \\
\text { hampers any } \\
\text { environmental } \\
\text { hazard } \\
\text { evaluation }\end{array}$ \\
\hline
\end{tabular}

The authors apologize to readers for these errors. 